\title{
Molecular cytogenetic analyses of breakpoints in apparently balanced reciprocal translocations carried by phenotypically normal individuals
}

\author{
Julia Baptista*1,2 ${ }^{*}$ Elena Prigmore ${ }^{3}$, Susan M Gribble ${ }^{3}$, Patricia A Jacobs ${ }^{1,2}$, Nigel P Carter ${ }^{3}$ \\ and John A Crolla ${ }^{1,2}$
}

\begin{abstract}
${ }^{1}$ Wessex Regional Genetics Laboratory, Salisbury District Hospital, Salisbury, Wiltshire, UK; ${ }^{2}$ Division of Human Genetics, School of Medicine, University of Southampton, Southampton, UK; ${ }^{3}$ The Wellcome Trust Sanger Institute, Wellcome Trust Genome Campus, Hinxton, Cambridge, UK
\end{abstract}

To test the hypothesis that translocation breakpoints in normal individuals are simple and do not disrupt genes, we characterised the breakpoints in 13 phenotypically normal individuals incidentally ascertained with an apparently balanced reciprocal translocation. Cases were karyotyped, and the breakpoints were refined by fluorescence in situ hybridisation until breakpoint-spanning clones were identified. $1 \mathrm{Mb}$ arrayCGH was performed as a whole genome analysis tool to detect any imbalances in chromatin not directly involved in the breakpoints. Breakpoint-associated imbalances were not found in any of the patients analysed in this study. However, breakpoints which disrupted known genes were identified in two patients, with RYR2 disrupted in one patient and COL13A1 in the other. In a further eight patients, Ensembl mapping data suggested that a gene might be disrupted by a breakpoint. In one further patient, the translocation was shown to be nonreciprocal. This study shows that apparently balanced reciprocal translocations in phenotypically normal patients do not have imbalances at the breakpoints, in contrast to phenotypically abnormal patients where the translocation breakpoints are often associated with cryptic imbalances. However, phenotypically normal individuals, and phenotypically abnormal individuals may have genes disrupted and therefore inactivated by one of the breakpoints. The significance of these disruptions remains to be determined.

European Journal of Human Genetics (2005) 13, 1205-1212. doi:10.1038/sj.ejhg.5201488;

published online 24 August 2005

Keywords: apparently balanced translocation; fluorescence in situ hybridisation; array-CGH

\section{Introduction}

Reciprocal translocations are one of the most common structural chromosome abnormalities in our species and are present in one in 500 unselected newborns studied with a moderate level of banding. ${ }^{1}$ This type of rearrangement is characterised by a two-way exchange of the segment distal

*Correspondence: J Baptista, Wessex Regional Genetics Laboratory, Salisbury, Wiltshire, SP2 8B], UK.

Tel: + 441722 429080; Fax: + 441722 338095;

E-mail: Julia.baptista@salisbury.nhs.uk

Received 11 May 2005; revised 19 July 2005; accepted 21 July 2005; published online 24 August 2005 to the breakpoint in each of the chromosomes involved and it is considered balanced when there is no apparent gain or loss of chromosome material. ${ }^{2}$

Balanced translocations usually segregate in families without phenotypic effects, although carriers may have reproductive difficulties, including recurrent miscarriages and/or offspring with congenital abnormalities due to segregation of an unbalanced form of the translocation. However, a proportion of translocation carriers are ascertained through a proband with a de novo balanced rearrangement. Such de novo translocations have been shown to have an increased risk of phenotypic abnormality 
with a frequency of $\sim 6 \% .^{3}$ In cases where a balanced reciprocal translocation is found in association with a patient presenting with a clinically abnormal phenotype this is generally postulated to be due to: (i) the rearrangement not being balanced but associated with a cryptic duplication or deletion at one or both breakpoints only detectable using molecular techniques; ${ }^{4-6}$ (ii) the rearrangement being balanced but one or more breakpoints have interrupted or modulated the expression of a gene(s), which is causal to the phenotype; ${ }^{5,7}$ (iii) the association with the abnormal phenotype being due to chance.

The present study supplements recent publications in which cryptic imbalances at or close to the breakpoints in individuals with abnormal phenotypes were identified. ${ }^{4,6}$ Furthermore, in one study, cryptic imbalances were found in regions of chromosomes not involved in the translocations. ${ }^{6}$ We characterised the breakpoints in 13 phenotypically normal patients with a balanced translocation using molecular cytogenetic techniques and compared the analyses of the translocations in this phenotypically normal group of patients to those reported in phenotypically abnormal patients using identical techniques. Our hypothesis was that the translocation breakpoints would not have associated imbalances nor interrupt genes in contrast to those in the abnormal group.

\section{Methods}

\section{Study population}

Table 1 summarises the cell lines identification numbers, karyotypes and mode of ascertainment of the 13 patients analysed. They were all ascertained during routine clinical cytogenetics studies in the Wessex Regional Genetics Laboratory (WRGL). Our study was restricted to patients carrying an apparently balanced reciprocal translocation with a clinically normal phenotype where chromosomes of both parents had been investigated, and from which a lymphoblastoid cell line (LBC) had been established from at least the proband or one of the carrier family members. At the outset, it was decided that no clinical follow-up investigations would be requested for individuals who were clinically normal. Consequently, prior to all analyses, the LBCs and any associated samples of peripheral blood lymphocytes and/or DNA, were fully and irreversibly anonymised.

\section{Cell lines}

LBCs were established by Epstein-Barr virus (EBV) transformation, using standard methods. The cell lines were transformed by the European Cell and Culture Collection (ECACC) and deposited with both the ECACC and the WRGL cell bank. Case 8 was transformed and deposited only at the WRGL Cell Bank.

\section{Cytogenetic analysis}

Metaphase chromosomes were prepared from peripheral blood using standard methods. Karyotypes were determined by analysis of G-banded chromosomes at a minimum resolution of $550-600$ bands.

\section{FISH analysis}

Cell suspensions in 3:1 methanol:acetic acid were prepared from the cell lines and used in all the FISH experiments undertaken. The probes used in the FISH studies were derived from either BACs (Bacterial Artificial Chromosome) or PACs (P1-derived Artificial Chromosome). Probes mapping to the region of the cytogenetically determined

Table 1 Conventional karyotypes and ascertainment of the study population

\begin{tabular}{|c|c|c|c|}
\hline Case no. & Cell line Id & Karyotype & Ascertainment \\
\hline 2 & DD0505 & $46, X X, t(11 ; 17)(\mathrm{p} 13 ; \mathrm{p} 13.1) \mathrm{mat}$ & $\begin{array}{l}\text { Parent of child with developmental delay and karyotype } \\
46, X Y, t(11 ; 17)(p 13 ; p 13.1)\end{array}$ \\
\hline 3 & DD1319 & $46, X X, t(6 ; 22)(p 21.3 ; q 13) p a t$ & Parent of child with $46, \mathrm{XX}$, del(15)(q11q12) de novo \\
\hline 4 & DD1407 & $46, X X, t(7 ; 16)(p 15 ; q 22) m a t$ & Recurrent miscarriages \\
\hline 5 & DD0845 & $46, X Y, t(8 ; 16)(q 22.1 ; q 13) p a t$ & $\begin{array}{l}\text { Sibling of patient with puberty delay and karyotype } \\
46, X X, t(8 ; 16)(q 22.1 ; q 13)\end{array}$ \\
\hline 8 & LN2/3B03 & $46, X X, t(9 ; 20)(\mathrm{p} 24.1 ; \mathrm{p} 11.2 ? 3) \mathrm{mat}$ & $\begin{array}{l}\text { Parent of child with renal abnormalities and VSD with } \\
\text { karyotype } 46, X Y \operatorname{der}(9) \mathrm{t}(9 ; 20)(\mathrm{p} 24.1 ; \mathrm{p} 11.2 ? 3)\end{array}$ \\
\hline 9 & DD0182 & $46, X X, t(5 ; 18)(p 13 ; q 11) p a t$ & $\begin{array}{l}\text { Relative of } 46, \mathrm{XX}, \mathrm{t}(5 ; 18)(\mathrm{p} 13 ; \mathrm{q} 11) \text { pat that requested } \\
\text { prenatal testing because of family history of Down syndrome }\end{array}$ \\
\hline 10 & DD0282 & $46, X Y, t(1 ; 11)(q 42.3 ; q 21) p a t$ & $\begin{array}{l}\text { Parent of } 46, \mathrm{XX}, \mathrm{t}(1 ; 11)(\mathrm{q} 42.3 ; \mathrm{q} 21) \text { ascertained at prenatal } \\
\text { diagnosis for family history of Down syndrome }\end{array}$ \\
\hline 11 & DD1277 & $46, X X, t(3 ; 10)(p 23 ; q 21.2) p a t$ & $\begin{array}{l}\text { Relative of child with developmental delay and karyotype } \\
46, X Y, t(3 ; 10)(p 23 ; q 21.2)\end{array}$ \\
\hline
\end{tabular}


breakpoints were selected and obtained from the Ensembl Human Genome Browser (http://www.ensembl. org/Homo_sapiens/cytoview) or the UCSC Human Genome Browser (www.genome.ucsc.edu). Probe DNA was extracted by alkaline lysis and labelled by nick translation using either digoxigenin-11-dUTP or biotin-16-dUTP. FISH was performed following standard methods. Biotin-labelled probes were detected with FITC (green fluorochrome) and digoxigenin-labelled probes with sheep anti-DigTRITC (red fluorochrome). The slides were analysed with a Carl Zeiss Axiophot epifluorescence microscope and images were captured using a cooled charged-coupled deviced camera with Applied Imaging MacProbe software. The Ensembl Human Genome Browser and the UCSC Human Genome Browser, both based on the Human NCBI build 35 , were used to determine the genes contained in each breakpoint-spanning clone identified.

\section{Array-CGH}

DNA microarrays for comparative genomic hybridisation were constructed and array-CGH was performed according to the method described by Fiegler et al. ${ }^{8}$

\section{Results}

The results of all the cases are summarised in Table 2 .

Case 1: 46,XX,t(2;7)(p23.3;p22.3)de novo.ish $\mathrm{t}(2 ; 7)(\mathrm{RP} 11-$ 288C18sp;RP5-887P4sp)

FISH analysis demonstrated that BAC RP11-288C18 was split by the chromosome 2 breakpoint. This BAC contains the gene STRN (striatin) which, therefore, may have been disrupted by the breakpoint. PAC RP5-887P4, which contains no known genes, spanned the chromosome 7 breakpoint.

Case 2: 46,XX,t(11;17)(p13;p13.1)mat.ish $\mathrm{t}(11 ; 17)(\mathrm{RP} 11-$ 2G8sp;CTD-3083J7sp)

BAC RP11-2G8, containing no known genes, spanned the chromosome 11 breakpoint. BAC CTD-3083J7 spanned the chromosome 17 breakpoint, which encompasses a predicted gene of unknown function, NM_014859.3, which is physically larger than the spanning clone and must therefore be disrupted by the breakpoint.

Case 3: 46,XX,t(6;22)(p21.3;q13)pat.ish t(6;22)(RP11249P15sp;RP1-127L4sp)

BAC RP11-249P15, containing no known genes, was identified as the breakpoint-spanning clone on chromosome 6. PAC RP1-127L4 spanned the chromosome 22 breakpoint, which contains and may therefore disrupt the SLC5A1 (sodium/glucose cotransporter) gene.

Case 4: 46,XX,t(7;16)(p15;q22)mat.ish $\mathrm{t}(7 ; 16)(\mathrm{RP} 11-$ 842N18sp;RP11-419C5sp)

BAC RP11-842N18, containing no known genes, spanned the chromosome 7 breakpoint. BAC RP11-419C5 spanned the chromosome 16 breakpoint and contains the gene WWP2 (Nedd-4-like E3 ubiquitin-protein ligase
WWP2), and the predicted gene NP_001011880.1 (secretory protein LOC348174).

Case 5: 46,XY,t(8;16)(q22.1;q13)pat.ish $\mathrm{t}(8 ; 16)(\mathrm{RP} 11-$ 410L14sp;RP11-486F10sp)

BAC RP11-410L14, containing the ORS2 (Oxysterol binding protein-related protein 2) gene and the $\mathrm{COH} 1$ (Cohen syndrome protein 1) gene, was split by the chromosome 8 breakpoint. The BAC RP11-486F10, containing no known genes, spanned the chromosome 16 breakpoint.

Case 6: 46, XX, t(1;19)(q42.13;p13.2)mat.ish $\mathrm{t}(1 ; 19)(\mathrm{RP} 11-$ 757H9sp;CTB-55O6sp)

BAC RP11-757H9 was identified as the breakpointspanning BAC on chromosome 1 and contains the $L B R$ (Lamin B receptor) gene. BAC CTB-55O6 spanned the chromosome 19 breakpoint and contains the genes RFX1 (MHC class II regulatory factor), RLN3 (Relaxin 3 precurSor), IL27RA (class I cytokine receptor), SAMD1 (sterile alpha motif domain containing 1), PRKACA (cAMP-dependent protein kinase, alpha-catalytic subunit), ASF1B (ASF1 anti-silencing function 1 homolog B), LPHN1 (Latrophilin 1 precursor) and the predicted genes of unknown function: Q9HAAO_HUMAN, XP_292820.4, XP_053966.3, and $X P \_058967.10$, which may be disrupted by the breakpoint.

Case 7: 46,XX,t(16;18)(q24;q21.1)mat.ish t(16;18)(RP11517C16sp;RP11-980C16sp)

BAC RP11-517C16 was found to be split by the chromosome 16 breakpoint. This BAC contains two predicted genes: ATC4_HUMAN (Probable calcium-transporting ATPase) and NP_065998.2 (unknown function). BAC RP11-980C16 was split by the chromosome 18 breakpoint. This BAC contains the genes KATNAL2 (katanin p60 subunit A-like 2), TCEB3B (transcription elongation factor B polypeptide 3B elongin A2), HDHD2 (haloacid dehalogenase-like hydrolase domain containing 2), IER3IP1 (immediate-early response 3 interacting protein 1 ) and the predicted gene Q9P171_HUMAN of unknown function.

Case 8: 46,XX,t(9;20)(p24.1;p11.2?3)mat.ish t(9;20)(RP11472F14sp;RP4-737E23sp)

RP11-472F14 spanned the chromosome 9 breakpoint. This BAC contains the genes: UHRF2 (Np95-like ring finger protein isoform a) and GLDC (glycine dehydrogenase) and the predicted gene C9orf38, of unknown function. The PAC RP4-737E23 was split by the chromosome 20 breakpoint and contains the gene C1QR1 (Complement component $\mathrm{C} 1 \mathrm{q}$ receptor precursor).

Case 9: 46,XX,t(5;18)(p13;q11)pat.ish $\mathrm{t}(5 ; 18)(\mathrm{RP} 11-$ 60A21sp;RP11-266N21sp)

RP11-60A21 was found to span the chromosome 5 breakpoint. This BAC contains the LIFR (Leukaemia inhibitory factor receptor precursor) gene and the predicted gene NP_877950.1, of unknown function, which may be disrupted by the breakpoint. The BAC RP11$266 \mathrm{~N} 21$, containing the ROCK1 (Rho-associated protein kinase 1) gene, spanned the chromosome 18 breakpoint. 
Table 2 Summary of the results

\begin{tabular}{|c|c|c|c|c|c|}
\hline Case no. & Original karyotypes & Breakpoint spanning clone & $\begin{array}{c}\text { Likelihood in } \\
\text { percent that a gene } \\
\text { is disrupted by the } \\
\text { breakpoint }\end{array}$ & $\begin{array}{l}\text { No of genes } \\
\text { within clone }\end{array}$ & $\begin{array}{l}\text { Genes that map } \\
\text { within the } \\
\text { breakpoint } \\
\text { spanning clone }\end{array}$ \\
\hline 2 & $46, \mathrm{XX}, \mathrm{t}(11 ; 17)(\mathrm{p} 13 ; \mathrm{p} 13.1) \mathrm{mat}$ & $\begin{array}{l}\text { RP11-2G8 (11p12) } \\
\text { CTD-3083J7 (17p12) }\end{array}$ & $\begin{array}{r}0 \\
100\end{array}$ & $\begin{array}{l}0 \\
1\end{array}$ & NM_014859.3 \\
\hline 3 & $46, X X, t(6 ; 22)(p 21.3 ; q 13)$ pat & $\begin{array}{l}\text { RP11-249P15 (6p21.1) } \\
\text { RP1-127L4 (22q12.3) }\end{array}$ & $\begin{array}{l}0 \\
5\end{array}$ & $\begin{array}{l}0 \\
1\end{array}$ & SLC5A1 \\
\hline 4 & $46, X X, t(7 ; 16)(p 15 ; q 22) m a t$ & $\begin{array}{l}\text { RP11-842N18 (7p15.3) } \\
\text { RP11-419C5 (16q22.1) }\end{array}$ & $\begin{array}{r}0 \\
85\end{array}$ & $\begin{array}{l}0 \\
2\end{array}$ & $\begin{array}{l}\text { WWP2 } \\
\text { NP_001011880.1 }\end{array}$ \\
\hline 7 & $46, X X, t(16 ; 18)(q 24 ; q 21.1) m a t$ & $\begin{array}{l}\text { RP11-517C16 (16q24.1) } \\
\text { RP11-980C16 (18q21.1) }\end{array}$ & $\begin{array}{l}40 \\
60\end{array}$ & $\begin{array}{l}2 \\
5\end{array}$ & $\begin{array}{l}\text { AT2C2_HUMAN } \\
\text { NP_065998.2 } \\
\text { KATNAL2 } \\
\text { TCEB3B } \\
\text { HDHD2 } \\
\text { IER3IP1 } \\
\text { Q9P171_HUMAN }\end{array}$ \\
\hline 9 & $46, X X, t(5 ; 18)(p 13 ; q 11)$ pat & $\begin{array}{l}\text { RP11-60A21(5p13.2) } \\
\text { RP11-266N21 (18q11.1) }\end{array}$ & $\begin{array}{l}90 \\
70\end{array}$ & $\begin{array}{l}2 \\
1\end{array}$ & $\begin{array}{l}\text { NP_877950.1 } \\
\text { LIRF } \\
\text { ROCK1 }\end{array}$ \\
\hline 10 & $46, X Y, t(1 ; 11)(q 42.3 ; q 21) p a t$ & $\begin{array}{l}\text { RP11-214M7 (1q43) } \\
\text { RP11-99C10 (1 1q22.1) }\end{array}$ & $\begin{array}{r}100 \\
0\end{array}$ & $\begin{array}{l}1 \\
0\end{array}$ & RYR2 \\
\hline 11 & $46, X X, t(3 ; 10)(p 23 ; q 21.2) p a t$ & $\begin{array}{l}\text { RP11-123G15 (3p24.2) } \\
\text { RP11-548F9 (10q22.1) }\end{array}$ & $\begin{array}{l}100 \\
100\end{array}$ & $\begin{array}{l}1^{\mathrm{b}} \\
1\end{array}$ & $\begin{array}{l}\text { RARB } \\
\text { COL13A1 }\end{array}$ \\
\hline 12 & $46, \mathrm{XX}, \mathrm{t}(10 ; 18)(\mathrm{q} 24.3 ; \mathrm{q} 12.2) \mathrm{mat}$ & $\begin{array}{l}\text { RP11-163F15 (10q25.1) } \\
\text { RP11-142I20 (18q12.3) }\end{array}$ & $\begin{array}{l}0 \\
0\end{array}$ & $\begin{array}{l}0 \\
0\end{array}$ & \\
\hline 13 & $46, X Y, t(2 ; 3)(p 23.1 ; q 29) m a t$ & RP11-236C20 (2p23.3) & 0 & 0 & \\
\hline
\end{tabular}

Human NCBI build 35 .

${ }^{a}$ The physical size of the gene or genes mapping to a given BAC was calculated as a percent of the overall size of the BAC.

${ }^{\mathrm{b}} \mathrm{RP1} 1-123 \mathrm{G} 15$ is contained within the RARB gene, according to Ensembl. The UCSC Human Genome Browser maps RARB $200 \mathrm{~kb}$ downstream from RP11-123G15 and no know genes are contained within the BAC. 
Case 10: 46,XY,t(1;11)(q42.3;q21)pat.ish $\mathrm{t}(1 ; 11)(\mathrm{RP} 11-$ 214M7sp;RP11-99C10sp)

The BAC RP11-214M7 was split by the chromosome 1 breakpoint. This BAC contains part of the RYR2 (Ryanodine receptor 2) gene, which is physically larger than the spanning clone and must therefore be split by the breakpoint. BAC RP11-99C10, containing no known genes, spanned the chromosome 11 breakpoint.

Case 11: 46,XX,t(3;10)(p23;q21.2)pat.ish t(3;10)(RP11123G15sp;RP11-548F9sp)

FISH studies identified RP11-123G15 as the breakpointspanning clone on chromosome 3 and RP11-548F9 on chromosome 10 . The gene $R A R B$ (retinoic acid receptor) is also potentially disrupted by the breakpoint mapped within clone RP11-123G15. However, the physical mapping of $R A R B$ varies between Ensembl and UCSC browsers, so $R A R B$ either spans the BAC (Ensembl) and would be split by the breakpoint or maps $200 \mathrm{~kb}$ downstream of the BAC (UCSC) and would be unaffected. However, part of the gene COL13A1 (alpha 1 type XIII collagen isoform 1) is contained in RP11-548F9. As COL13A1 is physically larger than the breakpoint-spanning clone it must be split by the breakpoint (Figure $1 \mathrm{a}-\mathrm{d}$ ).

Case 12: 46,XX,t(10;18)(q24.3;q12.2)mat.ish t(10;18)(RP11163F15sp;RP11-142I20sp)

RP11-163F15 was identified as the breakpoint-spanning clone on chromosome 10 and RP11-142I20 on chromo- some 18. Neither of the BACs was found to contain any known genes.

Case 13: 46,XY,t $(2 ; 3)(\mathrm{p} 23.1 ; \mathrm{q} 29)$ mat.ish $\mathrm{t}(2 ; 3)$ (RP11236C20sp; 3qsubtel,+ 2 psubtel + )

BAC RP11-236C20, containing no known genes, spanned the chromosome 2 breakpoint. No reciprocal chromosome 3 product was found on the derived chromosome 2 and the derived chromosome 3 had retained its $3 \mathrm{q}$ subtelomere (196f4). Furthermore, the translocated 2p23. 22pter material, including the 2psubtelomere (2056f6) had translocated to distal 3q, showing that the translocation is nonreciprocal. FISH was also performed using probes for the subtelomeric regions of all chromosomes, which showed that no other chromosomes were involved in this rearrangement.

Array-CGH results: Array-CGH was performed using DNA extracted from the LBCs derived from all 13 patients. Only case 4 was found to have an imbalance, a deletion of $2.1 \mathrm{Mb}$ on chromosome 3 which was confirmed by FISH to be present in 70-90\% of the metaphases examined. The clones found to be deleted were: RP11-88B8 (3p21.31), RP1-289H18 (3p21.31), RP11-24C3 (3p21.31) and RP1178010 (3p21.31). PCR amplification of the microsatellite markers D3S3640, D3S643, D3S3629 and D3S1359 showed that the deletion was present in the cell line, but not in the genomic DNA, suggesting that the deletion is not constitutional and had occurred in vitro.
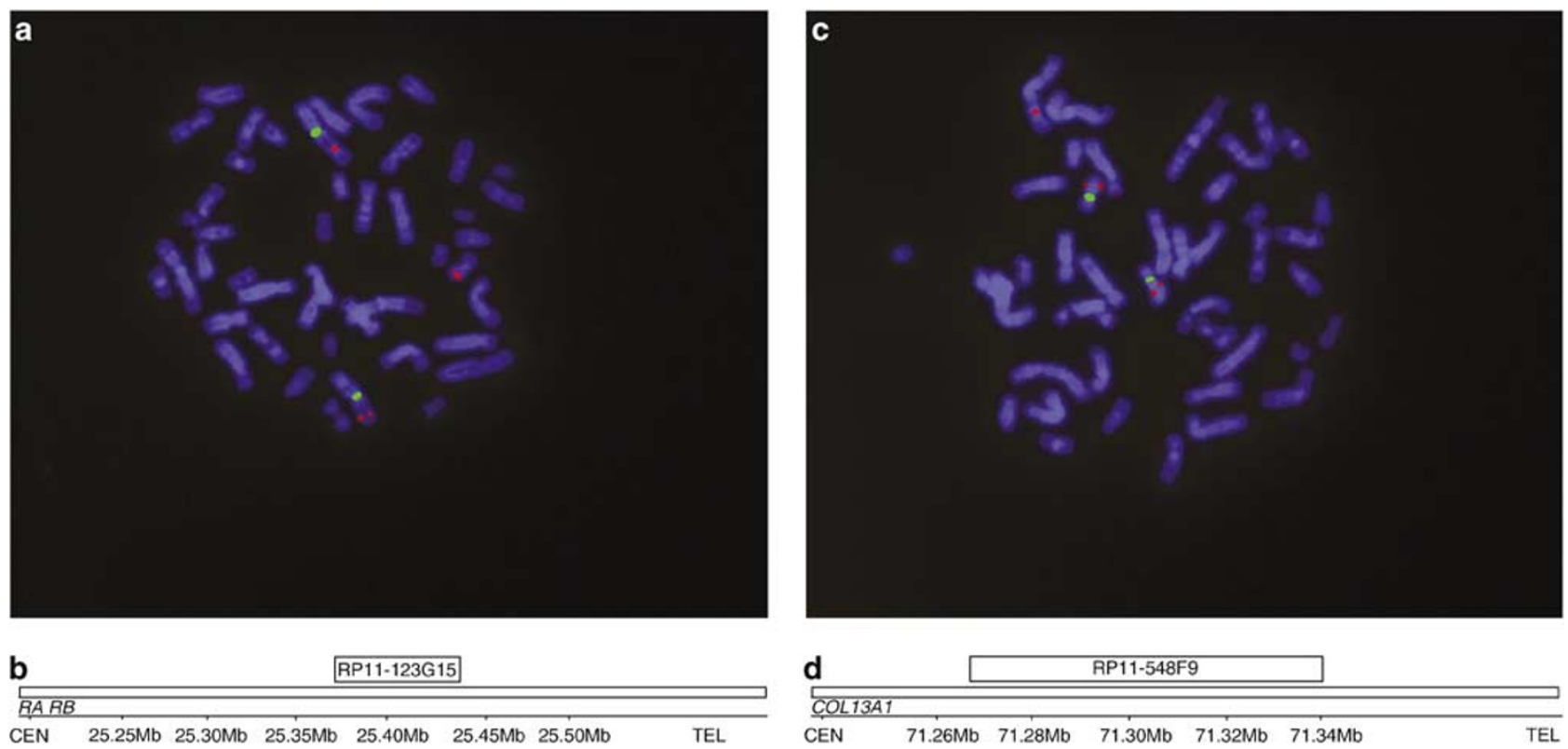

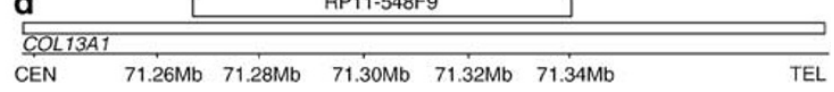

Figure 1 (a) Metaphase FISH in case 11. D3Z1 (green signal) maps to the chromosome 3 centromere. RP11-123G15 (red signal) spans the chromosome 3 breakpoint and hybridises to both $\operatorname{der}(3)$ and $\operatorname{der}(10)$. (b) Diagram adapted from www.ensembl.org showing that the chromosome 3 breakpoint-spanning clone in case 11, RP11-123G15, contains part of the RARB gene. (c) Metaphase FISH in case 11. D10Z1 (green signal) maps to the chromosome 10 centromere. RP11-548F9 (red signal) spans the chromosome 10 breakpoint and hybridises to both der(10) and der(3). (d) Diagram adapted from www.ensembl.org showing that the chromosome 10 breakpoint-spanning clone in case 11, RP11-548F9, contains part of the COL13A1 gene. 


\section{Discussion \\ No imbalances detected at the translocation breakpoints}

To the best of our knowledge, this study represents the first systematic analysis of translocation breakpoints found in clinically normal individuals. We found that the positions of the breakpoints in the normal individuals determined by using FISH analysis with BACs and PACs were comparable to the original cytogenetic locations. Furthermore, we were unable to find imbalances at the breakpoints at the level of resolution used (ie $\sim 150 \mathrm{~kb}$ ). These findings are in strong contrast to an equivalent study of clinically abnormal patients with apparently balanced translocations by Gribble et al, ${ }^{6}$ who uncovered previously unsuspected breakpoint complexity, imbalance near or at the breakpoints or genomic imbalance unrelated to the translocation in 6/10 cases. Similarly, Wirth et $\mathrm{al}^{4}$ found imbalances at breakpoints in 2/6 patients with apparently balanced chromosome rearrangement and mental retardation characterised by FISH. Astbury et $a l^{5}$ also reported cryptic deletions in 9/15 patients with a clinically abnormal phenotype and a de novo apparently balanced chromosome rearrangement. These observations support our hypothesis that breakpoints of normal individuals with apparently balanced chromosome rearrangements are relatively simple, whereas translocations in patients with clinically abnormal phenotypes are often complex and involve imbalance both at the breakpoint and/or elsewhere in the genome.

\section{A $2.1 \mathrm{Mb}$ imbalance detected by array-CGH}

Using array-CGH, Gribble et al ${ }^{6}$ found that 3/10 patients presented with imbalances in chromosome regions not involved in the translocations. We also applied $1 \mathrm{Mb}$ resolution array-CGH to our normal individuals and identified a deletion in 3p21.31 of approximately $2 \mathrm{Mb}$ in one case with 46,XX,t $(7 ; 16)(\mathrm{p} 15 ; \mathrm{q} 22)$ mat. However, further analysis showed that this imbalance was only present in all lymphoblastoid cell lines established from one blood sample and was absent from the patient's genomic DNA (peripheral blood). From this, we conclude that the deletion was an artefact of EBV transformation and does not reflect the patient's constitutional karyotype. Furthermore, the detection of imbalances in phenotypically abnormal carriers, but not in normal patients supports the importance of array-CGH in the routine investigation of apparently balanced translocations ascertained in patients with an abnormal clinical phenotype. ${ }^{6}$

\section{Genes associated with the breakpoints}

Our hypothesis was that the breakpoints of phenotypically normal patients with apparently balanced reciprocal translocation would not disrupt genes. However, the disruption of a recessive gene might occur, causing no phenotypic effect due to the presence of a normal allele on the homologous chromosome. Alternatively, the breakpoint might unmask a recessive gene if the allele on the normal homologue is mutated, although in our cohort there was no evidence for this mechanism. We found that no genes are disrupted in nine of 25 breakpoints analysed and that in 12 of 25 breakpoints, at least one gene colocalised with the breakpoint-spanning clone and so may be disrupted by the breakpoint. In the remaining three or four breakpoints (see Table 2), we were able to conclude that a gene would definitely have been split by the breakpoint as the gene was physically larger than the breakpoint-spanning clone.

\section{$R Y R 2$ is split by the breakpoint in $1 \mathrm{q} 42.3$}

Of particular interest is the gene $R Y R 2$, which codes for a cardiac muscle-type ryanodine receptor 2 which was split by the chromosome 1 breakpoint in case 10 . This receptor regulates excitation-contraction coupling by releasing $\mathrm{Ca}^{2+}$ ions from the sarcoplasmic reticulum. ${ }^{9}$ Priori et al ${ }^{10}$ identified four different missense mutations in $R Y R 2$ associated with catecholaminergic polymorphic ventricular tachycardia, a disorder characterised by stress-induced, bidirectional ventricular tachycardia in the absence of both structural heart disease and prolonged QT interval. Familial polymorphic ventricular tachycardia, an early onset inherited disease is also described as being caused by missense mutations in $R Y R 2^{11}$ as is arrythmogenic right ventricular cardiomyopathy type 2 , which is characterised by partial degeneration of the myocardium of the right ventricle, electrical instability and sudden death. ${ }^{12}$ The mutations described in all the above autosomal dominant disorders occurred in functionally important regions of the gene and involve highly conserved residues among the $R y R$ homologues from humans and other species. The importance of the RYR2 gene for normal heart function is supported by the observation that mice lacking $R Y R 2$ die early in embryonic life with heart abnormalities ${ }^{13}$ and evidence of decrease of RYR2 mRNA in failing human myocardium. ${ }^{14}$ However, we cannot speculate on the functional status of the gene in case 10 because the study design precludes us following up the current cardiac clinical history of the patient.

\section{$R A R B$ is potentially disrupted by the chromosome 3 breakpoint and COL13A1 is disrupted by the} chromosome 10 breakpoint of a $t(3 ; 10)(p 23 ; q 21.2)$ Our results suggested that $R A R B$ is potentially disrupted and COL13A1 is disrupted and therefore inactivated in case 11. An Ensembl transcript (ENST00000264330) was identified on chromosome 3, from position 25190893 to 25614424. According to Ensembl this transcript variant is from the $R A R B$ gene and the BAC RP11-123G15 is fully contained within this gene. However, the UCSC Human genome browser displays different $R A R B$ transcript variants starting $\sim 200 \mathrm{~kb}$ downstream from RP11-123G15, impli- 
cating that the gene is not disrupted by the breakpoint. Additional transcriptional analyses and protein function assays are necessary to elucidate the pattern of expression of the gene in this patient. $R A R B$ codes for a $\mathrm{B}$ retinoic acid receptor, a metabolite known to have morphogenic and teratogenic properties. The receptor controls cell function by directly regulating gene expression and it is a direct transcriptional target of Hoxb4, a transcription factor which is part of a developmental regulatory system that provides cells with specific positional identities on the anterior-posterior axis. ${ }^{15}$ Inappropriate expression of $R A R B$ in the liver is related to hepatocellular carcinogenesis $^{16}$ and its expression is selectively lost in premalignant oral lesions. ${ }^{17}$ Kreczet et al ${ }^{18}$ suggested that retinoids are involved in controlling the function of the dopaminergic mesolimbic pathway and that defects in retinoic acid receptor signalling may contribute to disorders such as Parkinson disease and schizophrenia. COL13A1 encodes the alpha-chain of type XIII collagen. Studies in mice expressing mutant Col13a1 suggest that type XIII collagen participates in the linkage between muscle fibre and basement membrane. ${ }^{19}$

\section{A nonreciprocal translocation}

In one of the translocations studied (case 13), conventional cytogenetic analysis reported the translocation to be reciprocal with distal breakpoints on both derivative chromosomes. However, further analysis using BAC FISH clearly showed that the rearrangement was nonreciprocal. This finding would therefore alter the reproductive risk for the carrier thereby illustrating the importance of molecular techniques to complement conventional cytogenetic analysis. Nonreciprocal translocations are characterised by a one-way exchange of chromosome material and are observed very rarely.

\section{Conclusions}

Our study has confirmed only part of our original hypothesis that the breakpoints of phenotypically normal patients with an apparently balanced reciprocal translocation are simple and do not disrupt genes. While we found that the majority of the translocation breakpoints were balanced (simple) we also found that in a surprisingly high proportion of breakpoints (15/25) a gene was either disrupted or possibly disrupted by the breakpoint. The level of resolution attained in this study was approximately $150 \mathrm{~kb}$, that is the average size of the genomic insert contained within the BACs used. It would be of interest to refine the breakpoints down to the sequence level and so confirm which genes (if any) are disrupted directly by the breakpoint. Higher resolution analyses might also identify small imbalances that remained undetected by the current methodologies.
Although we cannot determine the significance of the disruption of the genes observed, there is the possibility that these patients have gene defects that might increase their risk of developing late-onset disorders. Conversely, our findings could be of no clinical consequence. Additional studies, including long-term clinical follow-up of the patients might provide further insights on the loci involved and the possible effects of gene mutations of the type described here.

\section{Acknowledgements}

We thank Sarah Beal and Vivienne Maloney for help with the preparation of probes for FISH, and NS Thomas for performing microsatellite analysis. JB was supported by the Wellcome Trust.

\section{References}

1 Jacobs PA, Browne C, Gregson N, Joyce C, White H: Estimates of the frequency of chromosome abnormalities detectable in unselected newborns using moderate levels of banding. $J$ Med Genet 1992; 29: 103-108.

2 Gardner RJM, Sutherland GR: Chromosome Abnormalities and Genetic Counseling, 3rd ed Oxford: Oxford University Press, 2004; pp 36-59.

3 Warburton D: De novo balanced chromosome rearrangements and extra marker chromosomes identified at prenatal diagnosis: clinical significance and distribution of breakpoints. Am J Hum Genet 1991; 49: 995-1013.

4 Wirth J, Nothwang HG, van der Maarel S et al: Systematic characterisation of disease associated balanced chromosome rearrangements by FISH: cytogenetically and genetically anchored YACs identify microdeletions and candidate regions for mental retardation genes. J Med Genet 1999; 36: 271-278.

5 Astbury C, Christ LA, Aughton DJ et al: Detection of deletions in de novo 'balanced' chromosome rearrangements: further evidence for their role in phenotypic abnormalities. Genet Med 2004; 6: $81-89$

6 Gribble SM, Prigmore E, Burford DC et al: The complex nature of constitutional de novo apparently balanced translocations in patients presenting with abnormal phenotypes. I Med Genet 2005; 42: 8-16.

7 Giacalone JP, Francke U: Common sequence motifs at the rearrangement sites of a constitutional X/autosome translocation and associated deletion. Am J Hum Genet 1992; 50: 725-741.

8 Fiegler H, Carr P, Douglas EJ et al: DNA microarrays for comparative genomic hybridization based on DOP-PCR amplification of BAC and PAC clones. Genes Chromosomes Cancer 2003; 36: $361-374$.

9 Franzini-Armstrong C, Protasi F: Ryanodine receptors of striated muscles: a complex channel capable of multiple interactions. Physiol Rev 1997; 77: 699-729.

10 Priori SG, Napolitano C, Tiso N et al: Mutations in the cardiac ryanodine receptor gene (hRyR2) underlie catecholaminergic polymorphic ventricular tachycardia. Circulation 2001; 103: 196-200.

11 Laitinen PJ, Brown KM, Piippo K et al: Mutations of the cardiac ryanodine receptor (RyR2) gene in familial polymorphic ventricular tachycardia. Circulation 2001; 103: 485-490.

12 Tiso N, Stephan DA, Nava A et al: Identification of mutations in the cardiac ryanodine receptor gene in families affected with arrhythmogenic right ventricular cardiomyopathy type 2 (ARVD2). Hum Molec Genet 2001; 10: 189-194.

13 Takeshima H, Komazaki S, Hirose K, Nishi M, Noda T, Iino M Embryonic lethality and abnormal cardiac myocytes in mice lacking ryanodine receptor type 2. EMBO J 1998; 17: 3309-3316. 
14 Brillantes AM, Allen $\mathrm{P}$, Takahashi T, Izumo S, Marks AR: Differences in cardiac calcium release channel (ryanodine receptor) expression in myocardium from patients with endstage heart failure caused by ischemic versus dilated cardiomyopathy. Circ Res 1992; 71: 18-26.

15 Serpente P, Tumpel S, Ghyselinck NB et al: Direct crossregulation between retinoic acid receptor \{beta\} and Hox genes during hindbrain segmentation. Development 2005; 132: $503-513$.

16 de The $\mathrm{H}$, Marchio A, Tiollais P, Dejean A: A novel steroid thyroid hormone receptor-related gene inappropriately expres- sed in human hepatocellular carcinoma. Nature 1987; 330: $667-670$.

17 Lotan R, Xu XC, Lippman SM et al: Suppression of retinoic acid receptor-beta in premalignant oral lesions and its up-regulation by isotretinoin. N Eng J Med 1995; 332: 1405-1410.

18 Kreczel W, Ghyselinck N, Samad TA et al: Impaired locomotion and dopamine signalling in retinoid receptor mutant mice. Science 1998; 279: 863-867.

19 Kvist AP, Latvanlehto A, Sund M et al: Lack of cytosolic and transmembrane domains of type XIII collagen results in progressive myopathy. Am J Pathol 2001; 159: 1581-1592. 